\title{
THE CURVATURE OF GRADIENT RICCI SOLITONS
}

\author{
Ovidiu Munteanu and Mu-Tao Wang
}

\begin{abstract}
We study integral and pointwise bounds for the curvature of gradient shrinking Ricci solitons. As applications, we discuss gap and compactness results for gradient shrinkers.
\end{abstract}

\section{Introduction}

Gradient Ricci solitons are important in the study of Ricci flow, where they appear as possible singularity models. It is a central issue in the theory of Ricci flow to understand and classify these manifolds. Moreover, gradient Ricci solitons are natural extensions of Einstein manifolds, hence they are also interesting as a geometric partial differential equation.

An important and more studied class of gradient Ricci solitons are the gradient shrinkers. A gradient shrinking Ricci soliton is a Riemannian manifold $(M, g)$ for which there exists a potential function $f$ such that

$$
\operatorname{Ric}+\operatorname{Hess}(f)=\frac{1}{2} g,
$$

where Hess $(f)$ denotes the Hessian of $f$.

The classification of gradient shrinkers is known in dimensions $n=2$ and $n=3$, and assuming locally conformally flatness, in all dimensions $n \geq 4$ (see $[2,7,10,14-17$, 22]). Many of the techniques used in these works required some control of the Ricci curvature. For example, in [17], gradient shrinking Ricci solitons which are locally conformally flat were classified assuming an integral condition on the Ricci tensor

$$
\int_{M}|\operatorname{Ric}|^{2} e^{-f}<\infty
$$

This condition and other integral estimates of the curvature were later proved in [14]. Without making the assumption of being locally conformally flat, it is natural to ask whether similar estimates are true for the Riemann curvature tensor. In this paper we are able to prove pointwise estimates on the Riemann curvature, assuming in addition that the Ricci curvature is bounded. We will show that any gradient shrinking Ricci soliton with bounded Ricci curvature has Riemann curvature tensor growing at most polynomially in the distance function. We note that by Shi's local derivative estimates we can then obtain growth estimates on all derivatives of the curvature. This, in particular, proves weighted $L^{2}$ estimates for the Riemann curvature tensor and its covariant derivatives.

We point out that for self-shrinkers of the mean curvature flow Colding and Minicozzi [9] were able to prove weighted $L^{2}$ estimates for the second fundamental form,

Received by the editors August 11, 2010. 
assuming the mean curvature is positive. These estimates were instrumental in the classification of stable shrinkers. Our estimates can be viewed as parallel to theirs, however, the classification of gradient Ricci solitons is still a major open question in the field.

Before stating the results, let us set up the notation. An important observation is that the quantity $|\nabla f|^{2}+R-f$ is constant on $M,[11]$. Hence, by adding a constant to $f$ we can normalize it such that

$$
|\nabla f|^{2}+R=f
$$

In this paper, unless otherwise stated, we use the notation $(M, g, f)$ for a gradient shrinking Ricci soliton (1.1) with potential $f$ normalized as in (1.2). Let us denote with $|\mathrm{Ric}|$ and $|\mathrm{Rm}|$ the norms of the Ricci and Riemann tensors, respectively. We now state the main result of this paper.

Theorem 1.1. Let $(M, g, f)$ be a gradient shrinking Ricci soliton of dimension $n$ with bounded Ricci curvature

$$
|\mathrm{Ric}| \leq K \text { on } M \text {. }
$$

Then the Riemann curvature tensor grows at most polynomially in the distance function i.e., there exists a constant $a>0$ depending only on $n$ and $K$ and a constant $C>0$ so that

$$
|\operatorname{Rm}|(x) \leq C(r(x)+1)^{a},
$$

where $r(x):=d\left(x_{0}, x\right)$, for some fixed point $x_{0} \in M$.

We apply this theorem to prove a gap result for gradient shrinkers. We show that if the Ricci curvature is small enough everywhere on $M$ then the soliton is isometric to the Gaussian shrinker $\left(\mathbb{R}^{n}, d x^{2}, \frac{1}{4}|x|^{2}\right)$.

Corollary 1.1. Let $(M, g, f)$ be a gradient shrinking Ricci soliton. If $|\mathrm{Ric}| \leq \frac{1}{100 n}$ on $M$ then $M$ is isometric to the Gaussian soliton $\left(\mathbb{R}^{n}, d x^{2}, \frac{1}{4}|x|^{2}\right)$.

Yokota [20] has obtained a gap theorem for gradient shrinking Ricci solitons, namely he showed that if $(4 \pi)^{-\frac{n}{2}} \int_{M} e^{-f}>1-\varepsilon_{n}$ for some $\varepsilon_{n}$ depending on $n$ then $M$ is isometric to the Gaussian soliton.

Another application of our main theorem is in relation to compactness results for Ricci solitons. This topic has been recently studied in both compact $[4,19,21]$ and noncompact [12] settings. We recall a recent result for complete noncompact shrinkers, due to Haslhofer and Müller [12]. Let $\left(M_{i}, g_{i}, \bar{f}_{i}\right)$ a sequence of gradient shrinkers with the potentials $\bar{f}_{i}$ normalized such that $(4 \pi)^{-\frac{n}{2}} \int_{M_{i}} e^{-\bar{f}_{i}}=1$. Consider $z_{i}$ a point where $\bar{f}_{i}$ attains its minimum. Assume that Perelman's entropy $\mu_{i}$ has a uniform bound from below i.e., there exists $\bar{\mu}$ such that

$$
\mu_{i}:=(4 \pi)^{-\frac{n}{2}} \int_{M_{i}}\left(\left|\nabla \bar{f}_{i}\right|^{2}+R_{g_{i}}+\bar{f}_{i}-n\right) e^{-\bar{f}_{i}} \geq \bar{\mu} .
$$

If, moreover, for any $i$ and $r>0$

$$
\int_{B_{z_{i}}(r)}\left|\mathrm{Rm}_{g_{i}}\right|^{n / 2} \leq E(r)
$$


then a subsequence of $\left(M_{i}, g_{i}, \bar{f}_{i}, z_{i}\right)$ converges to an orbifold gradient shrinker in the pointed Cheeger-Gromov sense. Here $E(r)$ is a function depending on $r>0$ but independent of $i$.

As a consequence of our main theorem, we can show the following result. For this result it is not important how we normalize the function $f$.

Corollary 1.2. Let $(M, g, f)$ be a gradient shrinking Ricci soliton of dimension $n \geq 6$ and $|\mathrm{Ric}| \leq K$ on $M$. There exists a $r_{0}$ depending only on $n$ and $K$ such that if

$$
\int_{B_{x_{0}}\left(r_{0}\right)}|\mathrm{Rm}|^{n / 2} \leq L,
$$

for a minimum point $x_{0}$ of $f$, then for any $r>0$ we have

$$
\int_{B_{x_{0}}(r)}|\mathrm{Rm}|^{n / 2} \leq E(r),
$$

where $E(r)$ depends on $n, K, L$ and $r$.

In particular, Corollary 1.2 and the main theorem in [12] implies compactness of shrinkers assuming Ricci curvature bounds and only local curvature bounds.

Corollary 1.3. Let $\left(M_{i}, g_{i}, \bar{f}_{i}\right)$ be a sequence of gradient shrinking Ricci solitons normalized by $(4 \pi)^{-\frac{n}{2}} \int_{M_{i}} e^{-\bar{f}_{i}}=1$. Assume $\mu_{i} \geq \bar{\mu}$ and that we have a uniform bound on the Ricci curvature $\left|\operatorname{Ric}_{g_{i}}\right| \leq K$. Then there exists $r_{0}$ depending on $n$ and $K$ such that if

$$
\sup _{i} \int_{B_{z_{i}}\left(r_{0}\right)}\left|\mathrm{Rm}_{g_{i}}\right|^{n / 2}
$$

is finite for $z_{i}$ a minimum point of $\bar{f}_{i}$, then a subsequence of $\left(M_{i}, g_{i}, \bar{f}_{i}, z_{i}\right)$ converges to an orbifold gradient shrinker in the pointed Cheeger-Gromov sense.

\section{Proof of the curvature estimate}

The idea of the proof of Theorem 1.1 is the following. From the Ricci soliton equation (1.1), we can estimate

$$
\Delta_{f}|\mathrm{Rm}|^{2} \geq-c|\mathrm{Rm}|^{3},
$$

where $\Delta_{f}=\Delta-\nabla f \cdot \nabla$. It is natural therefore to attempt to use Moser iteration for this problem. Since the Ricci curvature is bounded below, the Sobolev constant is uniformly bounded on arbitrary balls of fixed radius $=1$. Therefore it is known that the Moser iteration will work if we can control the $L^{p}$ norm (for $p>n / 2$ ) of $|\mathrm{Rm}|$ on any ball of radius one. This is quite technical and it is done in Lemma 2.2 below.

To get $L^{p}$ estimates, we use the Ricci soliton equation and that the Ricci curvature is bounded. At the core of our estimates is a formula that relates the divergence of the Riemann curvature tensor of a Ricci soliton to the gradient of its Ricci curvature, see e.g., $[6,10]$

$$
\nabla_{l} R_{i j k l}=R_{i j k l} f_{l}=\nabla_{j} R_{i k}-\nabla_{i} R_{k j} .
$$

We first prove some Lemmas which are of independent interest. Everywhere in this section $(M, g, f)$ is a gradient shrinking Ricci soliton with bounded Ricci curvature. 
We use the notation:

$$
\begin{aligned}
|\mathrm{Ric}|^{2} & =\sum\left|R_{i j}\right|^{2}, \\
|\nabla \mathrm{Ric}|^{2} & =\sum\left|\nabla_{k} R_{i j}\right|^{2}, \\
|\mathrm{Rm}|^{2} & =\sum\left|R_{i j k l}\right|^{2}, \\
|\nabla \mathrm{Rm}|^{2} & =\sum\left|\nabla_{h} R_{i j k l}\right|^{2} .
\end{aligned}
$$

We recall some basic identities for gradient shrinking Ricci solitons, which are essential in the proof. First, taking trace of the soliton equation we get $R+\Delta f=\frac{n}{2}$. As mentioned in the introduction, using the Bianchi identities and normalizing $f$ we get $|\nabla f|^{2}+R=f$. This normalization of $f$ will be assumed throughout the paper. Other formulas that follow from Bianchi and Ricci identities and the soliton equation are $[10]$

$$
\begin{aligned}
\nabla_{i} R & =2 R_{i j} f_{j}, \\
\nabla_{k} R_{j k} & =R_{j k} f_{k}, \\
\nabla_{l} R_{i j k l} & =R_{i j k l} f_{l}, \\
\nabla_{j} R_{k i}-\nabla_{i} R_{k j} & =R_{i j k l} f_{l} .
\end{aligned}
$$

Lemma 2.1. Let $(M, g, f)$ be a gradient shrinking Ricci soliton with bounded Ricci curvature

$$
|\mathrm{Ric}| \leq K \text { on } M
$$

Then we have:

$$
\begin{aligned}
|\nabla \mathrm{Ric}|^{2} & \leq \frac{1}{2} \Delta|\mathrm{Ric}|^{2}-\frac{1}{2}\left\langle\nabla f, \nabla|\mathrm{Ric}|^{2}\right\rangle+2 K^{2}|\mathrm{Rm}| \\
|\nabla \mathrm{Rm}|^{2} & \leq \frac{1}{2} \Delta|\mathrm{Rm}|^{2}-\frac{1}{2}\left\langle\nabla f, \nabla|\mathrm{Rm}|^{2}\right\rangle+10|\mathrm{Rm}|^{3}
\end{aligned}
$$

Proof of Lemma 2.1. It is known (see e.g., [17]) that

$$
\begin{aligned}
& \Delta R_{i j}=\left\langle\nabla f, \nabla R_{i j}\right\rangle+R_{i j}-2 R_{i k j h} R_{k h} \\
& \Delta \mathrm{Rm}=\langle\nabla f, \nabla \mathrm{Rm}\rangle+\mathrm{Rm}-2\left(\mathrm{Rm}^{2}+\mathrm{Rm}^{\#}\right) .
\end{aligned}
$$

The lemma follows immediately from here, using the well known expressions for $\mathrm{Rm}^{2}$ and $\mathrm{Rm}^{\#}$.

We now prove the following estimate, which is of independent interest.

Lemma 2.2. Let $(M, g, f)$ be a gradient shrinking Ricci soliton of dimension $n$ with bounded Ricci curvature

$$
|\mathrm{Ric}| \leq K \text { on } M
$$

For any $p \geq 2$ there exists a positive constant $a>0$ depending only on $n, p$ and $K$ and a positive constant $C>0$ such that

$$
\int_{M}|\mathrm{Rm}|^{p}(f+1)^{-a} \leq C .
$$


In particular, there exist positive constants $C$ and a such that for any $x \in M$ we have:

$$
\int_{B_{x}(1)}|\mathrm{Rm}|^{p} \leq C(r(x)+1)^{2 a}
$$

where $r(x):=d\left(x_{0}, x\right)$ for a fixed point $x_{0} \in M$.

Proof of Lemma 2.2. Let us denote by $\rho:=2 \sqrt{f}$ and for $r>>1$ let

$$
D(r):=\{x \in M: \rho(x) \leq r\} .
$$

Notice that $D(r)$ is always compact, in fact approximates well the geodesic ball of radius $r$ when $r$ is large. Here we recall that $f$ has the following asymptotics, see [5]:

$$
\left(\frac{1}{2} r(x)-c\right)^{2} \leq f(x) \leq\left(\frac{1}{2} r(x)+c\right)^{2}, \text { for } r(x) \geq r_{0}
$$

Here and below we denote by $r(x)$ the distance from $x$ to a fixed point $x_{0} \in M$. Let us point out, moreover, that if $x_{0}$ is chosen to be a minimum point of $f$ then $c$ and $r_{0}$ will depend only on $n$, see [12]. We define the following cut-off

$$
\phi= \begin{cases}\frac{1}{r^{2}}\left(\frac{1}{4} r^{2}-f(x)\right), & \text { if } x \in D(r) \\ 0, & \text { if } x \in M \backslash D(r)\end{cases}
$$

Let $a$ be a fixed number to be determined later, depending on $n, K$ and $p$. Consider also $q$ a large enough integer, $q \geq 2 p+1$. We discuss first the case when $p \geq 3$, the case $2 \leq p<3$ will follow immediately by Hölder's inequality. We have, integrating by parts, that

$$
\begin{aligned}
a \int_{M}|\mathrm{Rm}|^{p}|\nabla f|^{2}(f+1)^{-a-1} \phi^{q}= & -\int_{M}|\operatorname{Rm}|^{p}\left\langle\nabla f, \nabla(f+1)^{-a}\right\rangle \phi^{q} \\
= & \int_{M}|\operatorname{Rm}|^{p}(\Delta f)(f+1)^{-a} \phi^{q} \\
& +\int_{M}|\operatorname{Rm}|^{p}(f+1)^{-a}\left\langle\nabla f, \nabla \phi^{q}\right\rangle \\
& +\int_{M}\left\langle\nabla|\operatorname{Rm}|^{p}, \nabla f\right\rangle(f+1)^{-a} \phi^{q} .
\end{aligned}
$$

The motivation for considering these functions in (2.4) is the following. We take $|\nabla f|^{2}$ because we want to use integration by parts and some symmetries of the Riemann curvature tensor (which are implied by the soliton equation). For shrinking solitons the factor $e^{-f}$ seems more convenient and natural than $(f+1)^{-a}$, however, the former factor only gives exponential growth control. Everywhere in this section $c$ will denote a constant that depends on $n, p, q$ and $K$ but not on $a$. We will use $C_{1}, C_{2}, \ldots$ etc. to denote finite constants that have a more complicated dependence, such as on 
$\sup _{\Omega}|\mathrm{Rm}|$ over some compact set $\Omega$. However, we stress that all the constants $c$ or $C_{1}, C_{2}, \ldots$ are independent of $r$. We now check that:

$$
a|\nabla f|^{2}(f+1)^{-a-1}-(\Delta f)(f+1)^{-a}=\left(\frac{a(f-R)}{f+1}-\left(\frac{n}{2}-R\right)\right)(f+1)^{-a} .
$$

Furthermore, since $R \geq 0$ on any gradient Ricci soliton ( $[3,8])$ we see that there exists a constant $r_{1}$ depending only on $n, K$ and $a$ (e.g., $\left.r_{1}=\sqrt{8 a(K+1)}\right)$ such that on $M \backslash D\left(r_{1}\right)$ we have

$$
a|\nabla f|^{2}(f+1)^{-a-1}-(\Delta f)(f+1)^{-a} \geq(a-n)(f+1)^{-a} .
$$

Notice also that by the choice of cut-off we have:

$$
\left\langle\nabla f, \nabla \phi^{q}\right\rangle=-\frac{q}{r^{2}} \phi^{q-1}|\nabla f|^{2} \leq 0
$$

Using these estimates in (2.4) it follows that:

$$
(a-n) \int_{M}|\mathrm{Rm}|^{p}(f+1)^{-a} \phi^{q} \leq \int_{M}\left\langle\nabla|\mathrm{Rm}|^{p}, \nabla f\right\rangle(f+1)^{-a} \phi^{q}+C_{1},
$$

where we have set

$$
C_{1}:=\int_{D\left(r_{1}\right)}\left(-a|\nabla f|^{2}(f+1)^{-1}+\Delta f+a-n\right)|\mathrm{Rm}|^{p}(f+1)^{-a} \phi^{q} .
$$

Let us compute, using the Bianchi identities:

$$
\begin{aligned}
\left\langle\nabla|\mathrm{Rm}|^{2}, \nabla f\right\rangle & =2 f_{h}\left(\nabla_{h} R_{i j k l}\right) R_{i j k l} \\
& =4 f_{h}\left(\nabla_{l} R_{i j k h}\right) R_{i j k l}
\end{aligned}
$$

Therefore, using this in the right-hand side of (2.5) we get

$$
\begin{aligned}
\int_{M} & \left\langle\nabla|\mathrm{Rm}|^{p}, \nabla f\right\rangle(f+1)^{-a} \phi^{q} \\
& =2 p \int_{M} f_{h}\left(\nabla_{l} R_{i j k h}\right) R_{i j k l}|\mathrm{Rm}|^{p-2}(f+1)^{-a} \phi^{q} \\
& =-2 p \int_{M} R_{i j k h} \nabla_{l}\left(f_{h} R_{i j k l}|\mathrm{Rm}|^{p-2}(f+1)^{-a} \phi^{q}\right) \\
& =I+I I+I I I+I V+V
\end{aligned}
$$

where

$$
\begin{aligned}
I & :=-2 p \int_{M} R_{i j k h} f_{h l} R_{i j k l}|\mathrm{Rm}|^{p-2}(f+1)^{-a} \phi^{q}, \\
I I & :=-2 p \int_{M} R_{i j k h} f_{h}\left(\nabla_{l} R_{i j k l}\right)|\mathrm{Rm}|^{p-2}(f+1)^{-a} \phi^{q},
\end{aligned}
$$

and

$$
\begin{aligned}
I I I & :=-2 p \int_{M} R_{i j k h} f_{h} R_{i j k l}\left(\nabla_{l}|\mathrm{Rm}|^{p-2}\right)(f+1)^{-a} \phi^{q}, \\
I V & :=2 a p \int_{M} R_{i j k h} f_{h} R_{i j k l} f_{l}|\mathrm{Rm}|^{p-2}(f+1)^{-a-1} \phi^{q} \\
V & :=-2 p q \int_{M} R_{i j k h} f_{h} R_{i j k l} \phi_{l}|\mathrm{Rm}|^{p-2}(f+1)^{-a} \phi^{q-1} .
\end{aligned}
$$


It is easy to see that since the Ricci curvature is bounded,

$$
I=-2 p \int_{M} R_{i j k h} f_{h l} R_{i j k l}|\mathrm{Rm}|^{p-2}(f+1)^{-a} \phi^{q} \leq c \int_{M}|\mathrm{Rm}|^{p}(f+1)^{-a} \phi^{q} .
$$

Furthermore, using that for a gradient shrinker we have (see e.g., [10])

$$
\nabla_{l} R_{i j k l}=R_{i j k l} f_{l}
$$

we see that

$$
I I+I V=-2 p \int_{M} R_{i j k h} f_{h} R_{i j k l} f_{l}|\mathrm{Rm}|^{p-2}\left(1-\frac{a}{f+1}\right)(f+1)^{-a} \phi^{q} \leq C_{2},
$$

where we have set

$$
C_{2}:=2 p \int_{D(2 \sqrt{a-1})}\left|R_{i j k h} f_{h}\right|^{2}|\mathrm{Rm}|^{p-2}\left(\frac{a}{f+1}-1\right)(f+1)^{-a} \phi^{q} .
$$

The estimate above follows because if $f(x)+1>a$ then the integral is negative. Let us point out, however, that in fact each of $|I I|$ and $|I V|$ can be estimated by a similar argument as in the proof of inequality (2.9) below. Clearly, since $a$ is independent of $r$, so are the constants $C_{1}$ and $C_{2}$ obtained so far. We use the above estimates in (2.5), and get that

$$
\begin{aligned}
&(a-c) \int_{M}|\mathrm{Rm}|^{p}(f+1)^{-a} \phi^{q} \\
& \leq-2 p \int_{M} R_{i j k h} f_{h} R_{i j k l}\left(\nabla_{l}|\mathrm{Rm}|^{p-2}\right)(f+1)^{-a} \phi^{q} \\
&+\frac{2 p q}{r^{2}} \int_{M}\left|R_{i j k h} f_{h}\right|^{2}|\mathrm{Rm}|^{p-2}(f+1)^{-a} \phi^{q-1}+C_{1}+C_{2} .
\end{aligned}
$$

Recall that for Ricci solitons we have (see e.g., $[6,10]$ )

$$
R_{i j k h} f_{h}=\nabla_{j} R_{i k}-\nabla_{i} R_{k j} .
$$

Then we can estimate the first term in the right-hand side of (2.6) by

$$
\begin{aligned}
- & 2 p \int_{M} R_{i j k h} f_{h} R_{i j k l}\left(\nabla_{l}|\mathrm{Rm}|^{p-2}\right)(f+1)^{-a} \phi^{q} \\
& \leq c \int_{M}|\nabla \mathrm{Ric}||\nabla \mathrm{Rm}||\mathrm{Rm}|^{p-2}(f+1)^{-a} \phi^{q} \\
& \leq c \int_{M}|\nabla \mathrm{Ric}|^{2}|\mathrm{Rm}|^{p-1}(f+1)^{-a} \phi^{q}+c \int_{M}|\nabla \mathrm{Rm}|^{2}|\mathrm{Rm}|^{p-3}(f+1)^{-a} \phi^{q} .
\end{aligned}
$$

We now work on the second term in the right-hand side of (2.6), and we will use below some interpolations which are important throughout the rest of the proof. We have,

$$
\begin{aligned}
\frac{1}{r^{2}} & \int_{M} R_{i j k h} f_{h} R_{i j k l} f_{l}|\mathrm{Rm}|^{p-2}(f+1)^{-a} \phi^{q-1} \\
& =\frac{2}{r^{2}} \int_{M}\left(\nabla_{j} R_{i k}\right) R_{i j k l} f_{l}|\mathrm{Rm}|^{p-2}(f+1)^{-a} \phi^{q-1} \\
& =-\frac{2}{r^{2}} \int_{M} R_{i k} \nabla_{j}\left(R_{i j k l} f_{l}|\mathrm{Rm}|^{p-2}(f+1)^{-a} \phi^{q-1}\right) \\
& =I+I I+I I I+I V+V
\end{aligned}
$$


where

$$
\begin{aligned}
I & :=-\frac{2}{r^{2}} \int_{M} R_{i k} f_{l j} R_{i j k l}|\mathrm{Rm}|^{p-2}(f+1)^{-a} \phi^{q-1}, \\
I I & :=-\frac{2}{r^{2}} \int_{M} R_{i k} f_{l}\left(\nabla_{j} R_{i j k l}\right)|\mathrm{Rm}|^{p-2}(f+1)^{-a} \phi^{q-1}, \\
I I I & :=-\frac{2}{r^{2}} \int_{M} R_{i k} R_{i j k l} f_{l}\left(\nabla_{j}|\mathrm{Rm}|^{p-2}\right)(f+1)^{-a} \phi^{q-1},
\end{aligned}
$$

and

$$
\begin{aligned}
I V & :=\frac{2 a}{r^{2}} \int_{M} R_{i k} R_{i j k l} f_{l} f_{j}|\mathrm{Rm}|^{p-2}(f+1)^{-a-1} \phi^{q-1}, \\
V & :=-\frac{2(q-1)}{r^{2}} \int_{M} R_{i k} R_{i j k l} f_{l} \phi_{j}|\mathrm{Rm}|^{p-2}(f+1)^{-a} \phi^{q-2} .
\end{aligned}
$$

Since the Ricci curvature is bounded, we get

$$
\begin{aligned}
I & =-\frac{2}{r^{2}} \int_{M} R_{i k} f_{l j} R_{i j k l}|\mathrm{Rm}|^{p-2}(f+1)^{-a} \phi^{q-1} \\
& \leq \frac{c}{r^{2}} \int_{M}|\mathrm{Rm}|^{p-1}(f+1)^{-a} \phi^{q-1} .
\end{aligned}
$$

Using again that

$$
\nabla_{l} R_{i j k l}=R_{i j k l} f_{l}
$$

and that $|\nabla f|^{2} \leq f \leq \frac{1}{4} r^{2}$ on $D(r)$, it follows:

$$
I I \leq c \int_{M}|\mathrm{Rm}|^{p-1}(f+1)^{-a} \phi^{q-1} .
$$

Furthermore,

$$
\begin{aligned}
I I I & =-\frac{2}{r^{2}} \int_{M} R_{i k} R_{i j k l} f_{l}\left(\nabla_{j}|\mathrm{Rm}|^{p-2}\right)(f+1)^{-a} \phi^{q-1} \\
& \leq c \int_{M}|\nabla \mathrm{Rm}||\mathrm{Rm}|^{p-2}(f+1)^{-a} \phi^{q-1} \\
& \leq c \int_{M}|\nabla \mathrm{Rm}|^{2}|\mathrm{Rm}|^{p-3}(f+1)^{-a} \phi^{q}+c \int_{M}|\mathrm{Rm}|^{p-1}(f+1)^{-a} \phi^{q-2} .
\end{aligned}
$$

Similarly,

$$
\begin{aligned}
I V & =\frac{2 a}{r^{2}} \int_{M} R_{i k} R_{i j k l} f_{l} f_{j}|\mathrm{Rm}|^{p-2}(f+1)^{-a-1} \phi^{q-1} \\
& \leq \frac{c a}{r^{2}} \int_{M}|\mathrm{Rm}|^{p-1}(f+1)^{-a} \phi^{q-1} \leq c \int_{M}|\mathrm{Rm}|^{p-1}(f+1)^{-a} \phi^{q-1},
\end{aligned}
$$

by taking $r \geq \sqrt{a}$. Finally, we also have

$$
V \leq \frac{c}{r^{2}} \int_{M}|\mathrm{Rm}|^{p-1}(f+1)^{-a} \phi^{q-2} .
$$


Using these estimates in $(2.8)$ we get

$$
\begin{aligned}
& \frac{2 p q}{r^{2}} \int_{M}\left|R_{i j k h} f_{h}\right|^{2}|\mathrm{Rm}|^{p-2}(f+1)^{-a} \phi^{q-1} \\
& \quad \leq c \int_{M}|\nabla \mathrm{Rm}|^{2}|\mathrm{Rm}|^{p-3}(f+1)^{-a} \phi^{q}+c \int_{M}|\mathrm{Rm}|^{p-1}(f+1)^{-a} \phi^{q-2} .
\end{aligned}
$$

Notice moreover that we can interpolate, using Young's inequality:

$$
\begin{aligned}
& \int_{M}|\operatorname{Rm}|^{p-1}(f+1)^{-a} \phi^{q-2} \\
& \quad \leq \int_{M}|\operatorname{Rm}|^{p-1} \phi^{q \frac{p-1}{p}} \phi^{\frac{q}{p}-2}(f+1)^{-a} \\
& \quad \leq \varepsilon \int_{M}|\operatorname{Rm}|^{p}(f+1)^{-a} \phi^{q}+c(\varepsilon) \int_{M}(f+1)^{-a} \phi^{q-2 p} .
\end{aligned}
$$

We want to use this in (2.9), hence here we can take $\varepsilon=1$. Moreover, to guarantee that $\phi^{q-2 p}$ is well defined, we take $q \geq 2 p+1$. We plug (2.10) in (2.9) and then use (2.9) and (2.7) in (2.6). It results that

$$
\begin{aligned}
(a-c) & \int_{M}|\mathrm{Rm}|^{p}(f+1)^{-a} \phi^{q} \\
\leq & c \int_{M}|\nabla \mathrm{Ric}|^{2}|\mathrm{Rm}|^{p-1}(f+1)^{-a} \phi^{q} \\
& +c \int_{M}|\nabla \mathrm{Rm}|^{2}|\mathrm{Rm}|^{p-3}(f+1)^{-a} \phi^{q}+C_{1}+C_{2}+C_{0} .
\end{aligned}
$$

We have denoted with

$$
C_{0}:=c \int_{M}(f+1)^{-a},
$$

and observe that taking $q=2 p+1$ and taking $a$ such that $a>\frac{n}{2}+1$ then $C_{0}$ is a finite constant, independent of $r$. Indeed it is known that the volume growth of $M$ is polynomial, see [5]

$$
\operatorname{Vol}\left(B_{x_{0}}(r)\right) \leq c r^{n}, \text { for all } r>0 \text {. }
$$

We finish the proof by estimating each of the two terms in the right-hand side of (2.11). Start with the first, which by (2.1) we have:

$$
\begin{aligned}
& 2 \int_{M}|\nabla \mathrm{Ric}|^{2}|\mathrm{Rm}|^{p-1}(f+1)^{-a} \phi^{q} \\
& \quad \leq \int_{M}\left(\Delta|\mathrm{Ric}|^{2}\right)|\mathrm{Rm}|^{p-1}(f+1)^{-a} \phi^{q} \\
& \quad-\int_{M}\left\langle\nabla f, \nabla|\mathrm{Ric}|^{2}\right\rangle|\operatorname{Rm}|^{p-1}(f+1)^{-a} \phi^{q}+c \int_{M}|\operatorname{Rm}|^{p}(f+1)^{-a} \phi^{q}
\end{aligned}
$$

Let us observe that

$$
\begin{aligned}
\int_{M} & \left(\Delta|\mathrm{Ric}|^{2}\right)|R m|^{p-1}(f+1)^{-a} \phi^{q} \\
= & -\int_{M}\left\langle\nabla|\operatorname{Ric}|^{2}, \nabla\left(|\operatorname{Rm}|^{p-1}(f+1)^{-a} \phi^{q}\right)\right\rangle \\
= & I+I I+I I I,
\end{aligned}
$$


where

$$
\begin{gathered}
I:=-\int_{M}\left\langle\nabla|\mathrm{Ric}|^{2}, \nabla|\mathrm{Rm}|^{p-1}\right\rangle(f+1)^{-a} \phi^{q} \\
I I:=a \int_{M}\left\langle\nabla|\mathrm{Ric}|^{2}, \nabla f\right\rangle|\mathrm{Rm}|^{p-1}(f+1)^{-a-1} \phi^{q} \\
I I I:=-q \int_{M}\left\langle\nabla|\operatorname{Ric}|^{2}, \nabla \phi\right\rangle|\operatorname{Rm}|^{p-1}(f+1)^{-a} \phi^{q-1} .
\end{gathered}
$$

It is easy to see that

$$
\begin{aligned}
I & \leq c \int_{M}|\nabla \mathrm{Ric}||\nabla \mathrm{Rm}||\mathrm{Rm}|^{p-2}(f+1)^{-a} \phi^{q}, \\
I I & \leq c a \int_{M}|\nabla \mathrm{Ric}||\mathrm{Rm}|^{p-1}(f+1)^{-a} \phi^{q}, \\
I I I & \leq \frac{c}{r} \int_{M}|\nabla \mathrm{Ric}||\mathrm{Rm}|^{p-1}(f+1)^{-a} \phi^{q-1} .
\end{aligned}
$$

Therefore, using this in (2.13) we get

$$
\begin{aligned}
\int_{M}\left(\Delta|\mathrm{Ric}|^{2}\right)|\mathrm{Rm}|^{p-1}(f+1)^{-a} \phi^{q} \leq & c \int_{M}|\nabla \mathrm{Ric}||\nabla \mathrm{Rm}||\mathrm{Rm}|^{p-2}(f+1)^{-a} \phi^{q} \\
& +c a \int_{M}|\nabla \mathrm{Ric}||\mathrm{Rm}|^{p-1}(f+1)^{-a} \phi^{q-1} .
\end{aligned}
$$

Furthermore, observe that

$$
\begin{aligned}
c \int_{M}|\nabla \mathrm{Ric}||\nabla \mathrm{Rm}||\mathrm{Rm}|^{p-2}(f+1)^{-a} \phi^{q} \leq & \frac{1}{4} \int_{M}|\nabla \mathrm{Ric}|^{2}|\mathrm{Rm}|^{p-1}(f+1)^{-a} \phi^{q} \\
& +c \int_{M}|\nabla \mathrm{Rm}|^{2}|\mathrm{Rm}|^{p-3}(f+1)^{-a} \phi^{q} .
\end{aligned}
$$

Similarly,

$$
\begin{aligned}
c a \int_{M}|\nabla \mathrm{Ric}||\mathrm{Rm}|^{p-1}(f+1)^{-a} \phi^{q-1} \leq & \frac{1}{4} \int_{M}|\nabla \mathrm{Ric}|^{2}|\mathrm{Rm}|^{p-1}(f+1)^{-a} \phi^{q} \\
& +c a^{2} \int_{M}|\mathrm{Rm}|^{p-1}(f+1)^{-a} \phi^{q-2} .
\end{aligned}
$$

We conclude from above that

$$
\begin{aligned}
\int_{M}\left(\Delta|\operatorname{Ric}|^{2}\right)|\mathrm{Rm}|^{p-1}(f+1)^{-a} \phi^{q} \leq & \frac{1}{2} \int_{M}|\nabla \operatorname{Ric}|^{2}|\mathrm{Rm}|^{p-1}(f+1)^{-a} \phi^{q} \\
& +c \int_{M}|\nabla \mathrm{Rm}|^{2}|\mathrm{Rm}|^{p-3}(f+1)^{-a} \phi^{q} \\
& +c a^{2} \int_{M}|\mathrm{Rm}|^{p-1}(f+1)^{-a} \phi^{q-2} .
\end{aligned}
$$


Moreover,

$$
\begin{aligned}
-\int_{M}\left\langle\nabla f, \nabla|\operatorname{Ric}|^{2}\right\rangle|\operatorname{Rm}|^{p-1}(f+1)^{-a} \phi^{q} & \leq c \int_{M}|\nabla \operatorname{Ric}||\operatorname{Rm}|^{p-1}|\nabla f|(f+1)^{-a} \phi^{q} \\
\leq & \frac{1}{2} \int_{M}|\nabla \operatorname{Ric}|^{2}|\operatorname{Rm}|^{p-1}(f+1)^{-a} \phi^{q} \\
& +c \int_{M}|\operatorname{Rm}|^{p-1}|\nabla f|^{2}(f+1)^{-a} \phi^{q} .
\end{aligned}
$$

We can use the same idea as in (2.10) to bound:

$$
\begin{aligned}
\int_{M}|\mathrm{Rm}|^{p-1}|\nabla f|^{2}(f+1)^{-a} \phi^{q} \leq & c \int_{M}|\mathrm{Rm}|^{p}(f+1)^{-a} \phi^{q} \\
& +c \int_{M}(f+1)^{-a}|\nabla f|^{2 p} \phi^{q} .
\end{aligned}
$$

Once again, we take $a$ large enough e.g., $a>\frac{n}{2}+p+1$ so that

$$
\int_{M}(f+1)^{-a}|\nabla f|^{2 p}<\int_{M}(f+1)^{-a+p}<\infty .
$$

We also use (2.10) for $\varepsilon=\frac{1}{a^{2}}$ to get

$$
\begin{aligned}
c a^{2} \int_{M}|\mathrm{Rm}|^{p-1}(f+1)^{-a} \phi^{q-2} \leq & c \int_{M}|\mathrm{Rm}|^{p}(f+1)^{-a} \phi^{q} \\
& +c a^{2 p} \int_{M}(f+1)^{-a} \phi^{q-2 p} .
\end{aligned}
$$

Therefore, plugging all these estimates in (2.12) implies

$$
\begin{aligned}
\int_{M}|\nabla \mathrm{Ric}|^{2}|\mathrm{Rm}|^{p-1}(f+1)^{-a} \phi^{q} \leq & c \int_{M}|\nabla \mathrm{Rm}|^{2}|\mathrm{Rm}|^{p-3}(f+1)^{-a} \phi^{q} \\
& +c \int_{M}|\mathrm{Rm}|^{p}(f+1)^{-a} \phi^{q} \\
& +c a^{2 p} \int_{M}(f+1)^{-a+p}
\end{aligned}
$$

By (2.11), this yields

$$
(a-c) \int_{M}|\mathrm{Rm}|^{p}(f+1)^{-a} \phi^{q} \leq c \int_{M}|\nabla \mathrm{Rm}|^{2}|\mathrm{Rm}|^{p-3}(f+1)^{-a} \phi^{q}+C
$$

where

$$
\begin{aligned}
C & :=C_{1}+C_{2}+C_{3}, \\
C_{3} & :=c a^{2 p} \int_{M}(f+1)^{-a+p} .
\end{aligned}
$$


Finally, let us use (2.2) to check that

$$
\begin{aligned}
2 \int_{M}|\nabla \mathrm{Rm}|^{2}|\mathrm{Rm}|^{p-3}(f+1)^{-a} \phi^{q} \\
\leq \int_{M}\left(\Delta|\mathrm{Rm}|^{2}\right)|\mathrm{Rm}|^{p-3}(f+1)^{-a} \phi^{q} \\
\quad-\int_{M}\left\langle\nabla f, \nabla|\mathrm{Rm}|^{2}\right\rangle|\mathrm{Rm}|^{p-3}(f+1)^{-a} \phi^{q} \\
\quad+c \int_{M}|\mathrm{Rm}|^{p}(f+1)^{-a} \phi^{q} .
\end{aligned}
$$

The first term in the formula above is

$$
\begin{aligned}
\int_{M} & \left(\Delta|\mathrm{Rm}|^{2}\right)|\mathrm{Rm}|^{p-3}(f+1)^{-a} \phi^{q} \\
= & -\int_{M}\left\langle\nabla|\mathrm{Rm}|^{2}, \nabla|\mathrm{Rm}|^{p-3}\right\rangle(f+1)^{-a} \phi^{q} \\
& +a \int_{M}\left\langle\nabla|\mathrm{Rm}|^{2}, \nabla f\right\rangle|\mathrm{Rm}|^{p-3}(f+1)^{-a-1} \phi^{q} \\
& -q \int_{M}\left\langle\nabla|\mathrm{Rm}|^{2}, \nabla \phi\right\rangle|\mathrm{Rm}|^{p-3}(f+1)^{-a} \phi^{q-1} .
\end{aligned}
$$

Since $p \geq 3$ it is clear that

$$
-\int_{M}\left\langle\nabla|\mathrm{Rm}|^{2}, \nabla|\mathrm{Rm}|^{p-3}\right\rangle(f+1)^{-a} \phi^{q} \leq 0 .
$$

On the other hand,

$$
\begin{aligned}
a \int_{M}\left\langle\nabla|\mathrm{Rm}|^{2}, \nabla f\right\rangle|\mathrm{Rm}|^{p-3}(f+1)^{-a-1} \phi^{q} & \leq a c \int_{M}|\nabla \mathrm{Rm}||\mathrm{Rm}|^{p-2}(f+1)^{-a} \phi^{q} \\
\leq & \frac{1}{4} \int_{M}|\nabla \mathrm{Rm}|^{2}|\mathrm{Rm}|^{p-3}(f+1)^{-a} \phi^{q} \\
& +c a^{2} \int_{M}|\mathrm{Rm}|^{p-1}(f+1)^{-a} \phi^{q} .
\end{aligned}
$$

Similarly we find:

$$
\begin{aligned}
& -q \int_{M}\left\langle\nabla|\mathrm{Rm}|^{2}, \nabla \phi\right\rangle|\mathrm{Rm}|^{p-3}(f+1)^{-a} \phi^{q-1} \\
& \quad \leq \frac{1}{4} \int_{M}|\nabla \mathrm{Rm}|^{2}|\mathrm{Rm}|^{p-3}(f+1)^{-a} \phi^{q}+c \int_{M}|\mathrm{Rm}|^{p-1}(f+1)^{-a} \phi^{q-2} .
\end{aligned}
$$

Notice, moreover, that

$$
\begin{aligned}
-\int_{M}\left\langle\nabla f, \nabla|\mathrm{Rm}|^{2}\right\rangle|\mathrm{Rm}|^{p-3}(f+1)^{-a} \phi^{q} & \leq 2 \int_{M}|\nabla \mathrm{Rm}||\nabla f||\mathrm{Rm}|^{p-2}(f+1)^{-a} \phi^{q} \\
\leq & \frac{1}{2} \int_{M}|\nabla \mathrm{Rm}|^{2}|\mathrm{Rm}|^{p-3}(f+1)^{-a} \phi^{q} \\
& +c \int_{M}|\mathrm{Rm}|^{p-1}|\nabla f|^{2}(f+1)^{-a} \phi^{q} .
\end{aligned}
$$


Using these estimates in (2.15) we get:

$$
\begin{aligned}
\int_{M}|\nabla \mathrm{Rm}|^{2}|\mathrm{Rm}|^{p-3}(f+1)^{-a} \phi^{q} \leq & c \int_{M}|\mathrm{Rm}|^{p}(f+1)^{-a} \phi^{q} \\
& +c a^{2} \int_{M}|\mathrm{Rm}|^{p-1}(f+1)^{-a+1} \phi^{q-2} .
\end{aligned}
$$

From interpolation as in formula (2.10), for $\varepsilon=\frac{1}{a^{2}}$, it follows:

$$
\begin{aligned}
c a^{2} \int_{M}|\mathrm{Rm}|^{p-1}(f+1)^{-a+1} \phi^{q-2} \leq & c \int_{M}|\mathrm{Rm}|^{p}(f+1)^{-a} \phi^{q} \\
& +c a^{2 p} \int_{M}(f+1)^{-a+p} \phi^{q-2 p} .
\end{aligned}
$$

Therefore, we have proved:

$$
\int_{M}|\nabla \mathrm{Rm}|^{2}|\mathrm{Rm}|^{p-3}(f+1)^{-a} \phi^{q} \leq c \int_{M}|\mathrm{Rm}|^{p}(f+1)^{-a} \phi^{q}+c a^{2 p} \int_{M}(f+1)^{-a+p} .
$$

This, by (2.14), implies that

$$
(a-c) \int_{M}|\mathrm{Rm}|^{p}(f+1)^{-a} \phi^{q} \leq C .
$$

Recall that $c$ is a constant depending only on $n, p$ and $K$ while $a$ is a sufficiently large arbitrary number. This shows that there exists $a$ depending on $n, p$ and $K$ such that

$$
\int_{M}|\mathrm{Rm}|^{p}(f+1)^{-a} \phi^{q} \leq C .
$$

To conclude the proof of the Lemma notice that if

$$
\rho(x) \leq \frac{1}{2} r
$$

then

$$
\phi(x) \geq \frac{3}{16}
$$

hence this shows that

$$
\int_{D\left(\frac{1}{2} r\right)}|\mathrm{Rm}|^{p}(f+1)^{-a} \leq C .
$$

Since $C$ is independent of $r$, making $r \rightarrow \infty$ we get

$$
\int_{M}|\operatorname{Rm}|^{p}(f+1)^{-a} \leq C .
$$

Moreover, from $C=C_{1}+C_{2}+C_{3}$ and the expressions for these constants we see that in fact we have the estimate

$$
\int_{M}|\operatorname{Rm}|^{p}(f+1)^{-a} \leq c \int_{D\left(r_{0}\right)}|\operatorname{Rm}|^{p}(f+1)^{-a}+c \int_{M}(f+1)^{-a+p},
$$

where $r_{0}$ is a fixed number, depending on $n, p$ and $K$. 
This proves the Lemma in the case $p \geq 3$. We can estimate as in (2.10) to get the claim for any $p \geq 2$. The claim that

$$
\int_{B_{x}(1)}|\mathrm{Rm}|^{p} \leq C(r(x)+1)^{2 a}
$$

follows immediately from $(2.3)$.

Having Lemma 2.2 we can finish the proof of Theorem 1.1, using Moser iteration.

Proof of Theorem 1.1. From (2.2) we infer that

$$
\begin{aligned}
\Delta|\mathrm{Rm}|^{2} & \geq 2|\nabla \mathrm{Rm}|^{2}+\left\langle\nabla f, \nabla|\mathrm{Rm}|^{2}\right\rangle-c|\mathrm{Rm}|^{3} \\
& \geq-c\left(|\mathrm{Rm}|+|\nabla f|^{2}\right)|\mathrm{Rm}|^{2} .
\end{aligned}
$$

Therefore,

$$
\Delta|\mathrm{Rm}|^{2} \geq-u|\mathrm{Rm}|^{2}
$$

where we have denoted

$$
u:=c\left(|\operatorname{Rm}|+|\nabla f|^{2}\right) .
$$

Since the Ricci curvature is bounded below, there exists a uniform bound on the Sobolev constant of the ball $B_{x}(1)$. More exactly, for any $\varphi$ with support in $B_{x}(1)$ we have, by the Sobolev inequality in [18]:

$$
\left(\int_{B_{x}(1)} \varphi^{\frac{2 n}{n-2}}\right)^{\frac{n-2}{n}} \leq C_{S} \int_{B_{x}(1)}\left(|\nabla \varphi|^{2}+\varphi^{2}\right) .
$$

The constant $C_{S}$ depends only on $n, K$ and $\operatorname{Vol}\left(B_{x}(1)\right)$. As proved by Carillo and $\mathrm{Ni}$ in [1], there exists a constant $k>0$ so that

$$
\operatorname{Vol}\left(B_{x}(1)\right) \geq k>0 \text { for all } x \in M \text {. }
$$

The constant $k$ depends on $n, K$ and on Perelman's invariant

$$
\mu=-\log \left((4 \pi)^{-\frac{n}{2}} \int_{M} e^{-f}\right)<\infty .
$$

Then the standard Moser iteration, see [13], implies that

$$
|\mathrm{Rm}|^{2}(x) \leq A \int_{B_{x}(1)}|\mathrm{Rm}|^{2}
$$

where

$$
A:=C\left(\int_{B_{x}(1)} u^{n}+1\right)
$$

for a constant $C$ depending only on $n$ and $C_{S}$. Since we showed in Lemma 2.2 that $\int_{B_{x}(1)} u^{n}$ grows at most polynomially in $r(x)$, the theorem follows from here. 


\section{Gap and compactness theorems}

In this section, we prove the gap theorem and the compactness theorem of shrinking Ricci solitons, based on the estimates proved above. Since everywhere in this section the Ricci curvature is bounded, we can apply Theorem 1.1 to see that

$$
|\mathrm{Rm}|(x) \leq C(1+r(x))^{2 a} .
$$

We follow a similar argument as in Theorem 1.1, this time using the weight $e^{-f}$ and paying more attention to the dependence on the Ricci curvature bound. This simplifies considerably the computations. Of great importance in the argument are the following identities (see e.g., $[6,10]$ )

$$
\begin{gathered}
\nabla_{l}\left(R_{i j k l} e^{-f}\right)=0, \\
\nabla_{j}\left(R_{i j} e^{-f}\right)=0 .
\end{gathered}
$$

Clearly, we do not need to use a cut-off here, since all the curvature terms will be integrable with respect to $e^{-f}$, by Theorem 1.1. As in the previous section, we define

$$
K:=\sup _{M}|\operatorname{Ric}| \text {. }
$$

We also assume that $K>0$, since otherwise there is nothing to prove. We denote by

$$
\Delta_{f}=\Delta-\nabla f \cdot \nabla
$$

the weighted Laplacian. Notice that $\Delta_{f}$ is self adjoint with respect to the weighted volume $e^{-f} d v$.

Since

it follows that:

$$
\Delta_{f}(f)=\Delta f-|\nabla f|^{2}=\frac{n}{2}-f
$$

$$
\begin{aligned}
\int_{M}|\mathrm{Rm}|^{p}\left(f-\frac{n}{2}\right) e^{-f}= & -\int_{M}|\mathrm{Rm}|^{p} \Delta_{f}(f) e^{-f} \\
= & \int_{M}\left\langle\nabla f, \nabla|\mathrm{Rm}|^{p}\right\rangle e^{-f} \\
= & p \int_{M} \nabla_{h} R_{i j k l} R_{i j k l} f_{h}|\mathrm{Rm}|^{p-2} e^{-f} \\
= & 2 p \int_{M} \nabla_{l} R_{i j k h} R_{i j k l} f_{h}|\mathrm{Rm}|^{p-2} e^{-f} \\
= & -2 p \int_{M} R_{i j k h} f_{h l} R_{i j k l}|\mathrm{Rm}|^{p-2} e^{-f} \\
& -2 p \int_{M} R_{i j k h} f_{h} R_{i j k l} \nabla_{l}\left(|\mathrm{Rm}|^{p-2}\right) e^{-f}
\end{aligned}
$$

As in Theorem 1.1, we take an arbitrary $p \geq 3$. Using the soliton equation and the Ricci curvature bound $\mid$ Ric $\mid \leq K$, we find that

$$
\begin{aligned}
R_{i j k h} f_{h l} R_{i j k l} & =\frac{1}{2}|\mathrm{Rm}|^{2}-R_{i j k h} R_{i j k l} R_{h l} \\
& \geq\left(\frac{1}{2}-K\right)|\mathrm{Rm}|^{2} .
\end{aligned}
$$


We can also estimate

$$
\begin{aligned}
-2 p \int_{M} R_{i j k h} f_{h} R_{i j k l} \nabla_{l}\left(|\mathrm{Rm}|^{p-2}\right) e^{-f} & =-4 p \int_{M}\left(\nabla_{j} R_{i k}\right) R_{i j k l} \nabla_{l}\left(|\mathrm{Rm}|^{p-2}\right) e^{-f} \\
& \leq 4 p(p-2) \int_{M}|\nabla \operatorname{Ric}||\nabla| \mathrm{Rm}|||\mathrm{Rm}|^{p-2} e^{-f} .
\end{aligned}
$$

Then (3.1) shows that

$$
\begin{aligned}
& \int_{M}\left(f-\frac{n}{2}+p(1-2 K)\right)|\mathrm{Rm}|^{p} e^{-f} \\
& \quad \leq 4 p(p-2) \int_{M}|\nabla \mathrm{Ric}||\nabla| \mathrm{Rm}|||\mathrm{Rm}|^{p-2} e^{-f} .
\end{aligned}
$$

We estimate the right-hand side of (3.2) as follows:

$$
\begin{aligned}
2 \int_{M}|\nabla \mathrm{Ric}||\nabla| \mathrm{Rm}|||\mathrm{Rm}|^{p-2} e^{-f} \leq & \frac{1}{p K} \int_{M}|\nabla R i c|^{2}|\mathrm{Rm}|^{p-1} e^{-f} \\
& +p K \int_{M}|\nabla| \mathrm{Rm}||^{2}|\mathrm{Rm}|^{p-3} e^{-f} .
\end{aligned}
$$

Furthermore, by (2.1)

$$
\Delta_{f}|\mathrm{Ric}|^{2} \geq 2|\nabla \mathrm{Ric}|^{2}-4 K^{2}|\mathrm{Rm}| .
$$

Consequently,

$$
\begin{aligned}
\int_{M}|\nabla \mathrm{Ric}|^{2}|\mathrm{Rm}|^{p-1} e^{-f} & \leq \frac{1}{2} \int_{M}\left(\Delta_{f}|\mathrm{Ric}|^{2}\right)|\mathrm{Rm}|^{p-1} e^{-f}+2 K^{2} \int_{M}|\mathrm{Rm}|^{p} e^{-f} \\
& =-\frac{1}{2} \int_{M}\left\langle\nabla|\mathrm{Ric}|^{2}, \nabla|\mathrm{Rm}|^{p-1}\right\rangle e^{-f}+2 K^{2} \int_{M}|\mathrm{Rm}|^{p} e^{-f} \\
\leq & (p-1) K \int_{M}|\nabla \mathrm{Ric}||\nabla| \mathrm{Rm}|||\mathrm{Rm}|^{p-2} e^{-f} \\
& +2 K^{2} \int_{M}|\mathrm{Rm}|^{p} e^{-f}
\end{aligned}
$$

Using this in (3.3) we get

$$
\begin{aligned}
\int_{M}|\nabla \mathrm{Ric}||\nabla| \mathrm{Rm}|||\mathrm{Rm}|^{p-2} e^{-f} \leq & \frac{2 K}{p} \int_{M}|\mathrm{Rm}|^{p} e^{-f} \\
& +p K \int_{M}|\nabla| \mathrm{Rm}||^{2}|\mathrm{Rm}|^{p-3} e^{-f}
\end{aligned}
$$

Plugging into (3.2), this yields

$$
\begin{aligned}
& \int_{M}\left(f-\frac{n}{2}+p(1-10 K)\right)|\mathrm{Rm}|^{p} e^{-f} \\
& \quad \leq 4 p^{2}(p-2) K \int_{M}|\nabla| \mathrm{Rm}||^{2}|\mathrm{Rm}|^{p-3} e^{-f}
\end{aligned}
$$


Finally, from (2.2) we have:

$$
\begin{aligned}
2 \int_{M}|\nabla| \mathrm{Rm}||^{2}|\mathrm{Rm}|^{p-3} e^{-f} \leq & 2 \int_{M}|\nabla \mathrm{Rm}|^{2}|\mathrm{Rm}|^{p-3} e^{-f} \\
\leq & \int_{M}\left(\Delta_{f}|\mathrm{Rm}|^{2}\right)|\mathrm{Rm}|^{p-3} e^{-f} \\
& +20 \int_{M}|\mathrm{Rm}|^{p} e^{-f} .
\end{aligned}
$$

Integrating by parts and using that $p \geq 3$ we find

$$
\begin{aligned}
\int_{M}\left(\Delta_{f}|\mathrm{Rm}|^{2}\right)|\mathrm{Rm}|^{p-3} e^{-f} & =-\int_{M}\left\langle\nabla|\mathrm{Rm}|^{2}, \nabla|\mathrm{Rm}|^{p-3}\right\rangle e^{-f} \\
& =-2(p-3) \int_{M}|\nabla| \mathrm{Rm}||^{2}|\mathrm{Rm}|^{p-3} e^{-f} .
\end{aligned}
$$

Consequently, using this in (3.5) it follows that:

$$
\left.\int_{M}|\nabla| \mathrm{Rm}\right|^{2}|\mathrm{Rm}|^{p-3} e^{-f} \leq \frac{10}{p-2} \int_{M}|\mathrm{Rm}|^{p} e^{-f} .
$$

Therefore, from (3.4) we conclude that for $p \geq 3$ we have:

$$
\int_{M}\left(f-\frac{n}{2}+p(1-50 p K)\right)|\mathrm{Rm}|^{p} e^{-f} \leq 0 .
$$

We are now ready to prove Corollaries 1.1 and 1.2.

Proof of Corollary 1.1. Let us take $p=n$ in (3.6). We check from (3.6) that if $K \leq \frac{1}{100 n}$ then

$$
\int_{M} f|\mathrm{Rm}|^{n} e^{-f} \leq 0
$$

Recall that $f$ is normalized such that $|\nabla f|^{2}+R=f$. In particular, since any gradient shrinker has $R \geq 0$ (see $[3,8]$ ), it follows that $f \geq 0$. Thus the above inequality implies that $M$ is flat i.e., $(M, g, f)$ is the Gaussian soliton $\left(\mathbb{R}^{n}, d x^{2}, \frac{1}{4}|x|^{2}\right)$.

Proof of Corollary 1.2. We take $p=\frac{n}{2}$ in (3.6) to see that

$$
\int_{M}\left(f-15 n^{2} K\right)|\mathrm{Rm}|^{\frac{n}{2}} e^{-f} \leq 0 .
$$

We fix $x_{0}$ a point where $f$ achieves its minimum on $M$. Then we have (see $[5,12]$ )

$$
\frac{1}{4}\left[\left(d\left(x_{0}, x\right)-5 n\right)_{+}\right]^{2} \leq f(x) \leq \frac{1}{4}\left(d\left(x_{0}, x\right)+\sqrt{2 n}\right)^{2},
$$

where $a_{+}:=\max \{0, a\}$.

Let us set

$$
r_{0}:=6 n+\sqrt{60 n^{2} K}
$$


Using this in (3.7) we see that:

$$
\begin{aligned}
\int_{M \backslash B_{x_{0}}\left(r_{0}\right)}|\mathrm{Rm}|^{\frac{n}{2}} e^{-f} & \leq \int_{f \geq 15 n^{2} K+1}|\mathrm{Rm}|^{\frac{n}{2}} e^{-f} \\
& \leq \int_{f \geq 15 n^{2} K+1}\left(f-15 n^{2} K\right)|\mathrm{Rm}|^{\frac{n}{2}} e^{-f} \\
& \leq 15 n^{2} K \int_{f \leq 15 n^{2} K+1}|\mathrm{Rm}|^{\frac{n}{2}} e^{-f} \\
& \leq 15 n^{2} K \int_{B_{x_{0}}\left(r_{0}\right)}|\mathrm{Rm}|^{\frac{n}{2}} \leq 15 n^{2} K L
\end{aligned}
$$

Using again (3.8) shows that for any $r>0$

$$
\begin{aligned}
\int_{B_{x_{0}}(r)}|\mathrm{Rm}|^{\frac{n}{2}} & \leq E(r), \text { for } \\
E(r) & :=15 n^{2} K L e^{\frac{1}{4}(r+\sqrt{2 n})^{2}} .
\end{aligned}
$$

This proves Corollary 1.2.

\section{Acknowledgments}

The first author wishes to thank Ben Chow for stimulating discussions and encouragement. We also thank Huai-Dong Cao for his interest in this work.

The first named author has been partially supported by NSF grant no. DMS1005484. The second named author has been partially supported by NSF grant no. DMS-1105483.

\section{References}

[1] J. Carillo and L. Ni, Sharp logarithmic Sobolev inequalities on gradient solitons and applications, Comm. Anal. Geom. 17 (2009), 721-753.

[2] G. Catino and C. Mantegazza, Evolution of the Weyl tensor under the Ricci flow, to appear in Ann. Inst. Fourier.

[3] H. D. Cao, Recent progress on Ricci solitons, Adv. Lect. Math. 11(2) (2010), 1-38.

[4] H. D. Cao and N. Sesum, A compactness result for Kahler Ricci solitons, Adv. Math. 211 (2007), 794-818.

[5] H. D. Cao and D. Zhou, On complete gradient shrinking Ricci solitons, J. Differ. Geom. 85(2) (2010), 175-186.

[6] X. Cao, Compact gradient shrinking Ricci solitons with positive curvature operator, J. Geom. Anal. 17 (2007), 451-459.

[7] X. Cao, B. Wang and Z. Zhang, On locally conformally flat gradient shrinking Ricci solitons, Commun. Contemp. Math. 13(2) (2011), 1-14.

[8] B. L. Chen, Strong uniqueness of the Ricci flow, J. Differential Geom. 82(2) (2009), 362-382.

[9] T. Colding and W. Minicozzi II, Generic mean curvature flow I; generic singularities, arXiv:0908.3788.

[10] M. Eminenti, G. La Nave and C. Mantegazza, Ricci solitons: the equation point of view, Man. Math. 127 (2008), 345-367.

[11] R. Hamilton, The formation of singularities in the Ricci flow, Surv. Differ. Geom. 2 (1995), 7-136.

[12] R. Haslhofer and R. Müller, A compactness theorem for complete Ricci shrinkers, Geom. Funct. Anal. 21 (2011), 1091-1116.

[13] P. Li, Lecture notes on geometric analysis, available on http://math.uci.edu/ pli/.

[14] O. Munteanu and N. Sesum, On gradient Ricci solitons, to appear in J. Geom. Anal. 
[15] L. Ni and N. Wallach, On a classification of the gradient shrinking solitons, Math. Res. Lett. 15(5) (2008), 941-955.

[16] G. Perelman, Ricci flow with surgery on three manifolds, arXiv:math.DG/0303109.

[17] P. Petersen and W. Wylie, On the classification of gradient Ricci solitons, Geom. Topol. 14(4) (2010), 2277-2300.

[18] L. Saloff-Coste, Uniformly elliptic operators on Riemannian manifolds, J. Differ. Geom. 36 (1992), 417-450.

[19] B. Weber, Convergence of compact Ricci solitons, Int. Math. Res. Notices, 2011(1), 96-118.

[20] T. Yokota, Perelman's reduced volume and gap theorem for the Ricci flow, Comm. Anal. Geom. 17 (2009), 227-263.

[21] X. Zhang, Compactness theorems for gradient Ricci solitons, J. Geom. Phys. 56 (2006), 2481-2499.

[22] Z. H. Zhang, Gradient shrinking solitons with vanishing Weyl tensor, Pacific J. Math. 242(1) (2009), 189-200.

Department of Mathematics, Columbia University, New York, NY 10027, USA

E-mail address: omuntean@math.columbia.edu

Department of Mathematics, Columbia University, New York, NY 10027, USA

E-mail address: mtwang@math.columbia.edu 
